NOTAS 



\title{
LA EPIDEMIA DE MALARIA DE 1783-1786: NOTAS SOBRE LA INFLUENCIA DE ANOMALÍAS CLIMÁTICAS Y CAMBIOS DE USOS DEL SUELO EN LA SALUD HUMANA*
}

\author{
Pablo Giménez-Font \\ Instituto Universitario de Geografía \\ Universidad de Alicante
}

\section{RESUMEN}

La malaria es una enfermedad vectorial que sintetiza perfectamente, también desde una perspectiva histórica, las relaciones entre sociedad, salud y medio ambiente. De esta forma, las variables que determinan sus ciclos de avance y retroceso conforman un esquema de relaciones complejas entre condiciones ambientales, usos del suelo, densidades humanas y condiciones socio-sanitarias. Partiendo de las actuales proyecciones de comportamiento de la malaria en relación con el cambio climático, el presente artículo plantea un esquema interpretativo de las causas de la epidemia ocurrida en España entre 1783 y 1786, dentro de un contexto climático excepcional denominado Oscilación Maldá. Para ello, se realiza un estudio de caso para el óvalo central valenciano (zona hiperendémica) valorando las anomalías climáticas del momento, pero también los importantes procesos de deforestación y expansión del cultivo del arroz y los discursos médicos de control de la enfermedad, en unas condiciones de insalubridad y desnutrición generalizadas.

Palabras clave: malaria, clima histórico, usos del suelo, España, Valencia.

\section{ABSTRACT}

Malaria is a vector-transmitted disease which perfectly reflects relationships between society, health and the environment over time. The variables which determine the fluctuating disease cycles of malaria include a number of complex links between environmental conditions, land use, human population densities, and health and sanitary conditions. Based on current projections for the behaviour of malaria in relation

\footnotetext{
* Este artículo ha sido elaborado a partir de la ponencia «La naturaleza de los espacios húmedos y el riesgo de malaria en el siglo XVIII», presentada en el V Seminario de Historia y Clima: naturaleza, riesgo y desastre en la España Moderna y Contemporánea, celebrado en la Universidad de Alicante los días 7 y 8 de mayo de 2008.
} 
to climate change, this article proposes a model which can be used to interpret the causes of the epidemic that occurred in Spain between 1783 and 1786, within the context of the exceptional climatic phenomenon known as the Malda Anomaly. In order to draw up this model, a case study was carried out focusing on the Central Valencia Basin (hyperendemic zone), evaluating the climatic anomalies of the time, as well as the significant deforestation processes, the expansion of rice farming and the medical debate regarding the control of the disease in conditions that were generally unhealthy and plagued with malnutrition.

Key words: malaria, historical climate, land use, Spain, Valencia.

\section{Introducción}

La malaria es una de las epidemias más extendidas del planeta, con 106 países donde se considera endémica, y con estimaciones que hablan de entre 700.000 y 2,7 millones de víctimas al año, la mayoría de ellas localizadas en países del África subsahariana (Patz y Olson, 2006: 5635). Su distribución, actualmente ligada a países en vías de desarrollo, convierte a casi la mitad de la población mundial en potenciales hospedadores de los tipos de Plasmodium que parasitan varias especies de mosquito Anopheles. Dentro de esta virtualidad, la cifra de seres humanos expuestos ha crecido exponencialmente, aunque en términos espaciales la enfermedad ha sido progresivamente erradicada en gran parte de las latitudes medias del Hemisferio Norte, sur de Brasil, Sudáfrica y Australia.

No obstante, la malaria ha sido una enfermedad percibida y reconocida desde la antigüedad, y sobre ella se han basado algunas de las teorías básicas relativas a la interacción entre medio ambiente y ser humano, en este caso alrededor de la salud. Hipócrates (s.V aC.) ya planteaba la existencia de una constitución ambiental que otorgaba a cada lugar unas características particulares, de manera que, junto al régimen de vida propio, determinaba las condiciones de vida de los habitantes allí presentes. Los espacios húmedos, dentro de este esquema determinista, asumieron de inmediato una percepción negativa que está en la base de la cultura occidental, al relacionar las aguas estancadas con la formación de vapores mefíticos o miasmas nocivos para la salud. Esta teoría, llamada genéricamente ambientalista o miasmática, fue depurando dichos argumentos con aportaciones provenientes de la medicina, que tuvieron un incremento cualitativo durante los siglos XVII y XVIII con contribuciones de distintos autores (de la Boe, Lancisi, Arbuthnot, etc.) (Urteaga, 1993; Capel, 1999). Aunque los vapores eran los supuestos causantes de un gran número de enfermedades de etiología diversa, la principal era conocida como Paludismo (palus $-=$ charco), que en su referencia al agua estancada es sinónimo de Malaria (mal'aria = mal aire). También, haciendo mención a sus efectos, se hablaba de fiebres tercianas, fiebres cuartanas o calenturas intermitentes, debido a su característica manifestación regular. De igual manera, se hacía referencia a si se trataba de fiebres malignas o benignas, cuestión que hay que relacionar con la afección de un tipo de plasmodium u otro (generalmente $P$. falciparum o $P$. vivax).

Cuando en 1880 Laverman descubrió que el vector de la enfermedad era un mosquito, la medicina había alcanzado un grado tal de conocimiento sobre su comportamiento que, en algunos textos médicos, la mayoría de ellos basados en la experiencia acumulada, bastaría con cambiar las palabras «miasma» o «vapor» por la palabra «mosquito», para pensar que se estaba hablando de la misma cosa ${ }^{1}$. No obstante, la diferente causalidad tuvo influencias directas sobre teorías médicas y estrategias de control de la enfermedad: si el ambientalismo 
resaltaba la necesidad de trasformar las condiciones del medio —último causante de la enfermedad—, la irrupción de las corrientes bacteriológicas a finales del siglo XIX recalcó la necesidad de actuar sobre el individuo y su entorno inmediato. Se trataba de sensibles diferencias con influencias directas sobre la aplicación de distintas políticas preventivas sobre un territorio. En el caso de la malaria, sin embargo, la ausencia de vacunas eficaces sigue dando prioridad a las actuaciones sobre el vector (mosquito Anopheles, en su estado larvario) y sobre su hábitat (espacios acuáticos) como forma eficaz de control. En este sentido, se trata de una enfermedad que mantiene vivo el desafío, planteado por McNeill (1984), de llegar a entender las complejas vinculaciones entre la sociedad, la salud y el medio ecológico a lo largo de la historia.

\section{El riesgo de malaria: la complejidad de un esquema interpretativo}

La visualización de un mapa mundial de distribución de la malaria a lo largo del siglo XX (Hay et al., 2004:328), permite valorar la progresiva reducción de grandes áreas endémicas, especialmente a partir de la segunda mitad del siglo XX. Los programas efectivos de control vectorial (especialmente los desarrollados a partir de la aplicación del DDT), junto con las mejoras en la alimentación de la población y de las medidas sanitarias y asistenciales, se pueden señalar como las causas principales de la disminución espacial de territorios de riesgo, aunque no en número de población vulnerable.

Sin embargo, son más complejas las causas que explican el establecimiento de un área endémica (con los diferentes grados de endemicidad) y un área epidémica. Aquí convergen variables interconectadas que comienzan por las propias condiciones ambientales, relacionadas con el clima, la topografía e, incluso, la vegetación. Pero no solo hay que considerar los factores relacionados con los artrópodos transmisores, sino que la presencia del hombre complica los análisis al introducir sensibles diferencias relacionadas con el desarrollo económico y social, el tipo de poblamiento, las migraciones, los usos del suelo e, incluso, los discursos de control de la enfermedad.

La distribución de la población mundial y las proyecciones de crecimiento para ciertas zonas del planeta - especialmente en el continente africano- indican un incremento de la población expuesta a la enfermedad (Hay et al., 2004). A la malnutrición y la ausencia de políticas sanitarias eficaces, hay que unir una gestión poco eficiente del agua (p.e. Klinkenberg et al., 2002) o los efectos de diferentes usos del suelo (p.e. Barbieri, et al., 2005) que favorecen la proliferación vectorial. Por ejemplo, un crecimiento urbano que no contemple una evacuación eficaz de aguas y unas mínimas condiciones de higiene, favorece la transmisión de malaria, en conjunción con mayores densidades de hospedadores humanos en contacto con los mosquitos. De igual forma, un incremento de la superficie de regadío o de cultivos acuáticos como el arroz, puede favorecer la formación de criaderos si no se adoptan medidas oportunas.

1 El conocido fragmento de Masdevall, es representativo: «La experiencia constante nos ha hecho siempre ver, y en ello convienen todos los hombres sabios, que los países pantanosos, y en que las aguas subterráneas están muy cerca de la superficie de la Tierra, son los en que los hombres están más sujetos a padecer semejantes enfermedades, y esto principalmente si la Primavera ha sido muy lluviosa, y los ríos han salido de madre y de sus álveos; en cuyos casos si los calores fuertes del verano entran temprano, se corrompen las aguas pantanosas; exhalan varios vapores pútridos que corrompen la atmósfera, de la que así viciada se siguen con abundancia en dichas regiones calenturas continuas, regulares e intermitentes, las que regularmente se experimentan más en la clase de gente que por su modo de vivir se exponen y trabajan la mayor parte del día al sol, y que de noche toman el sereno» (Masdevall, 1786: 53-54). 


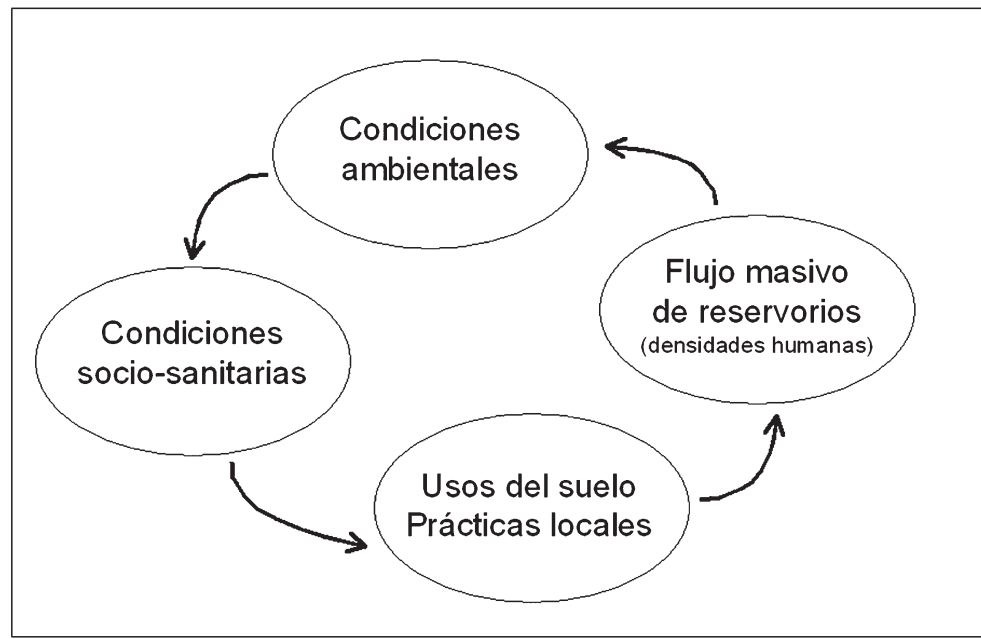

Figura 1. Variables generales que determinan los ciclos de avance de la malaria. Elaboración propia.

La desconcertante respuesta de la malaria a los fármacos y a las campañas de erradicación de tipo masivo, como las fumigaciones o la destrucción de ecosistemas húmedos, han despertado un nuevo interés por los métodos de control relacionados con la gestión ambiental. Se trata de medidas de costes reducidos, no tóxicas y sostenibles en el tiempo que inciden directamente en las prácticas locales y los usos del suelo de las áreas afectadas (Kreiser et al. 2005). Por otra parte, la mejora de las condiciones socio-sanitarias está directamente relacionada con el nivel de desarrollo económico de un país, al igual que las soluciones al problema del excesivo incremento demográfico.

Todos estos factores dependen, en última instancia, de elementos intrínsecos a la propia sociedad. Sin embargo, en los últimos años, la comunidad científica ha centrado parte de su interés en estudiar un factor como el clima - a priori, extrínseco al ser humano-, que puede determinar cambios en la biología y ecología de los vectores, los reservorios naturales y los hospedadores intermediarios (Reiter, 2001). No se trata únicamente de valorar la influencia a corto plazo de las variaciones de temperatura, precipitaciones o humedad, sino que se estudian los efectos de anomalías periódicas como el fenómeno ENSO (p.e. Koelle et al., 2005) o proyecciones de comportamiento a partir de las estimaciones de cambio climático a nivel global en un medio y largo plazo (Guiteko, et al., 2000; McMichael, et al. 2006).

Los cambios ambientales pueden afectar a la dinámica estacional y la distribución espacial de los mosquitos, modificando las relaciones entre éstos y los patógenos u hospedadores, y entre todos ellos y los reservorios. Con un incremento de la temperatura (hasta un máximo de $30-32^{\circ} \mathrm{C}$ ), se ha demostrado un aumento de la transmisibilidad de la malaria al darse las condiciones óptimas para la reproducción de las hembras y la maduración de las larvas. Junto a ello, un aumento de las precipitaciones puede favorecer la proliferación de criaderos (remansos de agua), que también pueden aparecer, en espacios húmedos, tras episodios de sequía (p.e., para el Este de Africa: Zhou et al. 2004).

Las estimaciones de cambio climático son poco esperanzadoras en relación con las enfermedades infecciosas y parasitarias. Para el caso de la malaria, se ha considerado un riesgo 
de dispersión sobre antiguas áreas endémicas, precisamente relacionado con la presencia de inviernos más lluviosos y cálidos y veranos más calurosos y secos. Para el territorio español, predicciones realizadas para el año 2050 presuponen escenarios de bajo riesgo que contrastan, por ejemplo, con los calculados para el SE de EEUU o de Brasil (Rogers y Randolph, 2000). Investigaciones más recientes, sin embargo, manifiestan la susceptibilidad de ciertas enfermedades vectoriales a los posibles escenarios de cambio climático proyectados para España, entre los que se encuentra el regreso - puntual, gracias a las condiciones socio-sanitarias del país - de la malaria (López-Vélez y Molina, 2005). En todo caso, la relación entre esta enfermedad y las variaciones en el clima es todavía una cuestión abierta que, por antigua, requiere también de perspectivas históricas y estudios detallados.

\section{Estudio de caso: la epidemia de malaria de 1783-1786 y las causas de proliferación vectorial en el antiguo Reino de Valencia}

Durante el siglo XVIII se produce en España un aumento cuantitativo y cualitativo de la información referida a epidemias y endemias de distinto signo. El brote infeccioso reproducido entre 1783 y 1786 marca, en este sentido, un verdadero hito en la historia de la medicina española: su virulencia fue tal, que la información contemporánea referida a sus consecuencias y las hipótesis sobre sus causas son muy abundantes, al igual que los posteriores estudios generales y regionales (en especial, Peset y Peset, 1972b; Pérez-Moreda, 1980 y 1985; Riera, 1980; Pérez-Medina, 1991; Alberola y Bernabé, 1999). A pesar de su comportamiento cíclico, la expansión del paludismo durante estos años ya no respondió a un simple crecimiento de la enfermedad por encima de sus tasas normales. La malaria desbordó sus límites naturales y, en unión con enfermedades de etiología difícil de precisar, gran parte de España se vio afectada por la epidemia. Pérez-Moreda (1980: 342) ofrece cifras superiores al millón de infectados y más de 100.000 fallecidos. Se ha considerado que la enfermedad empezó a expandirse en 1783 desde Lleida (Peset y Peset, 1972b: 74), pero es posible que un conjunto de condiciones favorables, principalmente de tipo climático, incrementaran el ámbito de actuación del mosquito desde espacios hiperendémicos, como los arrozales valencianos o los marjales cercanos a Cartagena. Después de afectar a numerosas poblaciones de Cataluña, Valencia o Murcia entre 1783 y 1785, durante 1786 la malaria produjo estragos en pueblos y ciudades de Andalucía (Sevilla, Córdoba, Málaga, Jaén), Toledo, La Alcarria y la Submeseta Norte (Pérez-Moreda, 1980: 337-339).

Un completo estudio de las consecuencias de la epidemia de 1784 en la comarca valenciana de l'Horta (que rodea la ciudad de Valencia) ofrece un reducido índice de mortalidad del $9 \%$ y una baja tasa de letalidad (29-37\%o), pero importantes tasas de morbilidad, en torno al 237\%o (Pérez-Medina, 1991: 146), que en algunos lugares supera el 500\%o e incluso el $1.000 \%$ (Peset y Peset, 1972a: 331). En el primer ejemplo, al tratarse mayoritariamente de las denominadas «tercianas benignas» (posiblemente producidas por Plasmodium vivax), 94 de cada 100 enfermos no morían directamente a causa de la infección, pero sus cuerpos quedaban debilitados para hacer frente a otro tipo de afecciones y para continuar con las labores agrícolas, que quedaban así desatendidas. En otros espacios, los informes médicos hacen referencia a la virulencia del paludismo, al que califican de «tercianas malignas» (producidas probablemente por Plasmodium falciparum) y, por tanto, mucho más mortales (Riera, 1980; Pérez-Moreda, 1985).

El valioso cuestionario enviado por las autoridades a diversos pueblos afectados en Valencia (Peset y Peset, 1972a: 327 y ss.), permite conocer, de la mano de los ayuntamientos y párrocos encuestados, alguna de las causas de la epidemia detectadas en ese mismo año de 1784. En primer lugar, se destacan las condiciones de pobreza en las que 


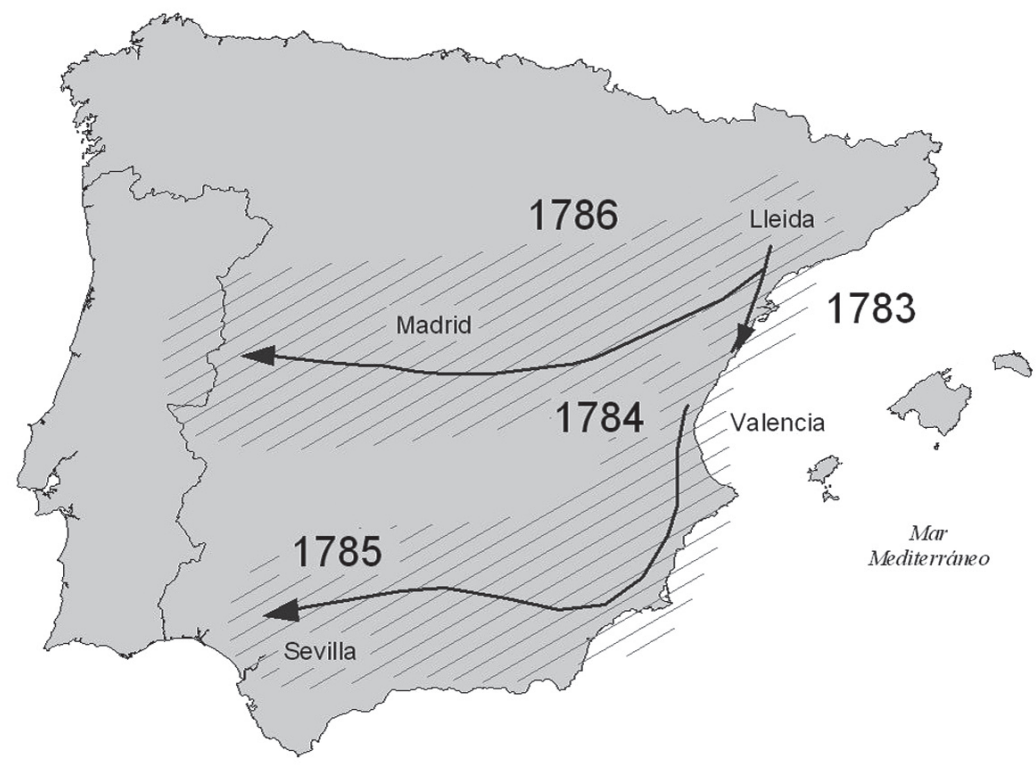

Figura 2. Avance de la epidemia en España, 1783-1786. Elaboración propia.

se encuentran los campesinos, en ocasiones con problemas de desnutrición y una excesiva carga de trabajo. En correspondencia con este hecho, las condiciones higiénicas eran muy deficientes, especialmente en pueblos pequeños donde el agua de consumo humano estaba contaminada por los arrozales, los estercoleros y los animales que convivían con el hombre dentro de las casas. Las aguas detenidas en balsas y acequias eran muy comunes dentro de los núcleos de población, pero en los espacios ribereños y llanuras aluviales como las de los ríos Xúquer y Túria, se destacaba especialmente la expansión de los arrozales. Todo ello se enmarcaba, además, en la presencia de unos años caracterizados por la inestabilidad atmosférica, que parecían haber favorecido la formación de espacios encharcados y los correspondientes miasmas.

Visualizando la figura 1, todos estos datos son variables del esquema que operan a distintos niveles jerárquicos. En el caso de las condiciones ambientales, las posibles oscilaciones climáticas y los cambios en los usos del suelo pueden repercutir de forma generalizada en la expansión de una enfermedad como la malaria, al influenciar directamente sobre la distribución de distintos tipos de vector (mosquitos Anopheles) y reservorios (seres humanos). Este hecho otorga validez a un estudio de caso histórico que puede clarificar algunas hipótesis de partida y establecer nuevas cuestiones en la determinación de los factores de riesgo asociados a la infección de malaria. 


\subsection{Cambios ambientales naturales: los efectos de la Oscilación 'Maldá'}

La epidemia de 1783-1786 se inscribe en una década muy sugerente desde el punto de vista de la climatología histórica, como mínimo, a nivel Europeo (Kington, 1988). Los crecientes estudios en esta rama de la climatología han determinado, para el Mediterráneo español, la presencia de tres grandes oscilaciones climáticas en los últimos 500 años (Barriendos y Martín-Vide, 1998), caracterizadas por un aumento de la frecuencia media de comportamientos extremos, relacionados con sequías e inundaciones. El episodio transcurrido entre, aproximadamente, 1760 y 1800, ha recibido el nombre de Oscilación Maldá (Barriendos y Llasat, 2003), verificándose la máxima intensidad de sequías, inundaciones y variaciones de la temperatura media entre los años 1775 y 1790. Las conclusiones de este estudio, elaborado a partir de proxy-data relativos a Cataluña, son extrapolables al sector occidental del Mediterráneo europeo, aunque todavía son necesarios trabajos de detalle en otros espacios.

Por ejemplo, una recopilación preliminar de proxy-data para el antiguo Reino de Valencia (tabla 1) parece confirmar la presencia de dicha oscilación, aunque sin un tratamiento cuantitativo ni aplicación de índices de intensidad en el contexto de varios siglos. Los datos utilizados provienen de documentación histórica y de un vaciado de bibliografía actual (p.e. Alberola, 2006). La mayoría de ellos se refiere a las consecuencias de episodios puntuales sobre infraestructuras o cultivos y sobre cosechas. Sin embargo, existen otros documentos (manuscritos e impresos) de carácter más genérico, que realizan valoraciones sobre la

Tabla 1

PROXY-DATA PARA LA DÉCADA DE 1780 EN EL ANTIGUO REINO DE VALENCIA

\begin{tabular}{|c|c|}
\hline Año & Tipo de episodio \\
\hline 1779 & $\begin{array}{c}\text { Sequía (> en Orihuela). Avenida Riu Serpis. Inundación } \\
\text { ríos Xúquer y Montesa }\end{array}$ \\
\hline 1780 & Rogativas pro-serenitate (Carcaixent) \\
\hline 1781 & Rogativas pro-serenitate (Carcaixent). Temporales \\
\hline 1782 & Lluvias intensas. Inundación del R. Xúquer \\
\hline 1783 & $\begin{array}{c}\text { Inundaciones ríos Xúquer, Túria, Palancia } \\
\text { Deslizamientos masivos en la Ribera Alta (Muntanya } \\
\text { Assolada, M. del Aixavegó) }\end{array}$ \\
\hline 1784 & $\begin{array}{c}\text { Rogativas pro-serenitate (Carcaixent) Inundación del R. } \\
\text { Xúquer }\end{array}$ \\
\hline 1785 & Inundación del R. Xúquer y Riu Albaida \\
\hline 1786 & Lluvias intensas. Inundación del R. Xúquer \\
\hline 1787 & Sequías, lluvias torrenciales y granizadas \\
\hline 1788 & Comienza un enfriamiento climático \\
\hline 1789 & Avenidas del Riu Monnegre y Xúquer-Albaida \\
\hline
\end{tabular}

Fuente: Base de Datos del Grupo de Investigación Historia y Clima (Universidad de Alicante). 
inestabilidad del tiempo atmosférico para una escala temporal más amplia, de varios años. Se trata de dietarios, informes técnicos y, especialmente, obras de ciencia realizadas por médicos, generalmente con alusiones claras a la relación entre esta epidemia y las condiciones del clima. Por ejemplo, para el conjunto de España, memoriales como los de García Burunda (1785), Villalba (1803), Tovares (1786), Masdevall para el caso catalán (1786), Troncoso para la ciudad de Córdoba (1785) o de Ased para Zaragoza (1784), ponen de manifiesto dicha correspondencia. García Burunda hacía referencia a la situación atmosférica de 1785 afirmando que «en todo el invierno y primavera fueron copiosas, abundantísimas las lluvias, y el estío ha sido seco y ardoroso» (cit. en Pérez-Moreda, 1985: 337), mientras que el mencionado de Ased (1784:3) escribe, refiriéndose al mismo año, que «a un Estío demasiadamente caliente y seco, han sucedido el Otoño, Invierno y Primavera tan abundantes de aguas, que han dado que discurrir à los físicos [...]». Pérez-Moreda (1980: 338 y ss.) ofrece numerosos testimonios locales en esta misma línea.

En el caso valenciano, las referencias son igualmente abundantes, especialmente relacionadas con el polémico cultivo del arroz y su discutida vinculación con el paludismo (vid. los numerosos textos transcritos por Peset y Peset, 1972a). Distintos informes y memoriales, realizados tanto desde la medicina y la administración política, como por parte de los poderosos movimientos pro y anti-arroceros, ofrecen información ambiental muy valiosa sobre la relación entre la epidemia y el clima de la década de 1780. Aunque todavía no se ha realizado un estudio sistemático de dicha información como proxy-data, desde un punto de vista cualitativo permite valorar la importancia de las anomalías climáticas ocurridas en aquel momento y profundizar en su conocimiento. La variabilidad atmosférica es la condición del esquema inicial con más posibilidades de reproducirse, en términos parecidos, en un corto o medio plazo.

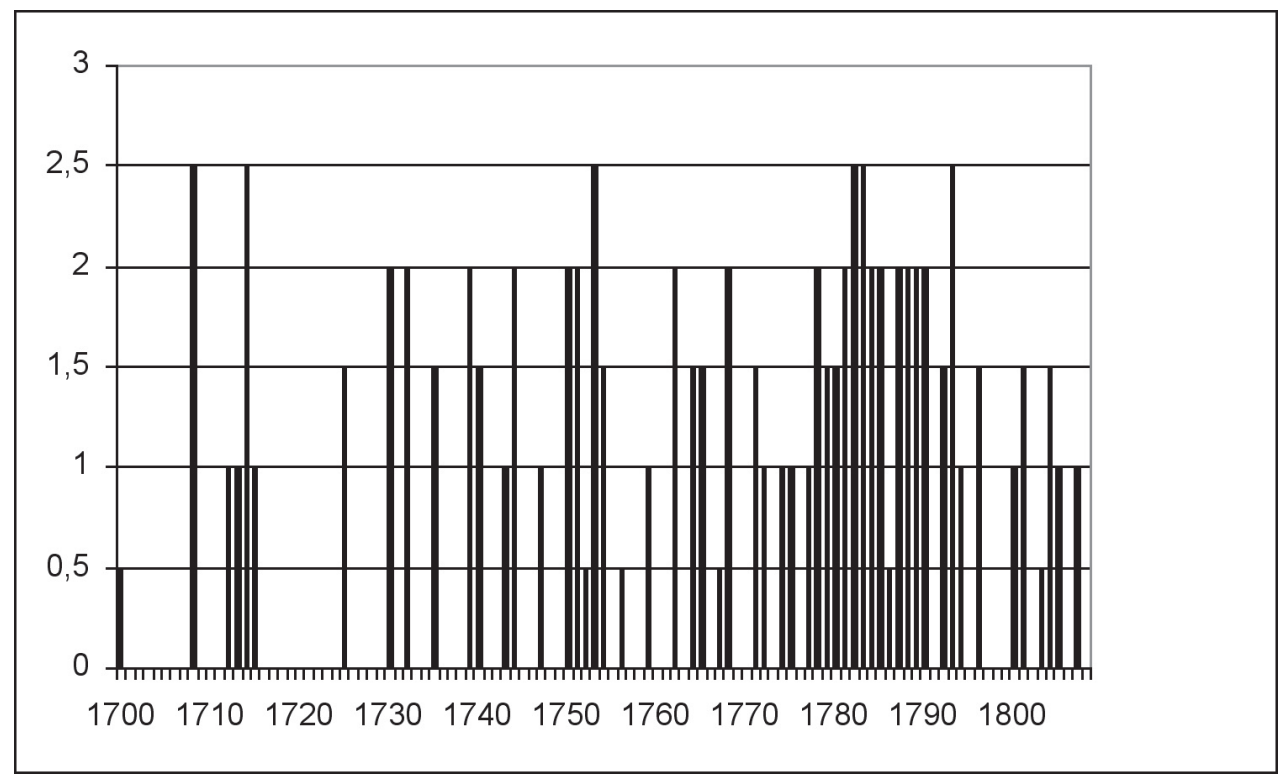

FIGURA 3. Índice preliminar de hidrometeoros aplicado a la Ribera del Xúquer (1700-1810) (valor medio de inundaciones, heladas, lluvias persistentes y granizo). Elaboración propia a partir de Peris (2001). 


\subsection{Cambios de usos del suelo y gestión hidráulica deficiente}

Junto a las condiciones climáticas particulares, la epidemia de la década de 1780 debe contextualizarse dentro de unas variables económicas y sociales ligadas a importantes cambios de usos del suelo y al crecimiento demográfico. Efectivamente, el siglo XVIII se ha caracterizado por intensos procesos roturadores, que en el óvalo central del antiguo Reino de Valencia encabezó el cultivo del arroz (Giménez-Font, 2008). Las condiciones particulares de esta gramínea, de elevados beneficios netos anuales, convirtieron su desarrollo en tierras valencianas en un verdadero problema de Estado, precisamente por su relación con el paludismo. El crecimiento de la superficie arrocera entre 1730 y 1807 (figura 4a) se produjo en paralelo a un recrudecimiento de la enfermedad, hasta el punto de comprometer la población de algunos lugares. El arroz alcanzó la condición de monocultivo en muchas zonas de la Ribera del Xúquer, cuando todavía no se habían producido las grandes desecaciones en la Albufera de Valencia (figura 4b). De esta manera, el avance del cultivo y de las superficies inundadas, se realizó sobre espacios húmedos, pero también sobre huertas y antiguos secanos de las riberas del Xúquer y Túria, vega de Xàtiva, Bajo Segura e, incluso, el río Algar (figura 5).

La teoría ambientalista o miasmática, y también la percepción popular, relacionó de inmediato la humedad ambiental y edáfica de los espacios arroceros con la presencia de fiebres intermitentes. En la Ribera del Xúquer, el incremento de las nieblas y los rocíos fue tal, que otras producciones agrarias se vieron seriamente comprometidas, favoreciendo una mayor expansión del cultivo del arroz. El papel del mismo en los procesos de condensación, en conjunción con avanzados estados de degradación forestal que, en Valencia, se aceleraron durante la Edad Moderna, tuvieron una influencia directa en los cambios en la interacción tierra-atmósfera. Aunque se trata de hipótesis por desarrollar, nos encontramos ante cambios ecológicos inducidos por el hombre que, a medio y corto plazo, presentan influencias directas sobre el comportamiento cíclico de enfermedades como la que nos ocupa.

El crecimiento de las superficies inundadas, y por tanto de los reservorios de vectores de la malaria, se acompañó de una gestión hidráulica poco eficaz y de prácticas locales favorecedoras del hábitat del mosquito. El incremento de los arrozales supuso importantes conflictos en el regadío y son comunes las quejas derivadas del malgasto de agua, fugas procedentes de acequias y encharcamientos producidos por esta causa. De igual manera, se encuentran con abundancia denuncias relativas al escaso mantenimiento de las parcelas de arroz y canales, favoreciendo la presencia de vegetación y la reproducción de molestos mosquitos: «observándose igualmente una total omisión en limpiar las aguas de los campos, que de propósito las dexan llenar de maleza para que no tengan fácil salida y rebalse á los campos, y acreditar por aquel medio de que son pantanosos: en cuya agua corrompida se crian ranas y otras sabandijas que muriéndose con el calor, aumentan la putrefacción é innumerable multitud de mosquitos, que aseguran los Curas ser capaces de acabar con el hombre mas alentado, si una sola noche quedara indefenso, causando notabilísimo perjuicio á los pobres enfermos, que se ven precisados á entrar luz en los quartos aun para el preciso alimento» (Memorial, 1803: 41v).

El arroz llegaba hasta las puertas de pueblos y ciudades, y la morbilidad producida por la malaria causaba, con altibajos, verdaderos estragos en la población. Los informes médicos aluden a las escasas condiciones higiénicas, a la relación de la enfermedad con la desnutrición, la lactancia o la vejez. Pero también se hacen interesantes reflexiones sobre la afluencia masiva de inmigrantes, es decir, flujos de reservorios que no estaban connaturalizados con el 

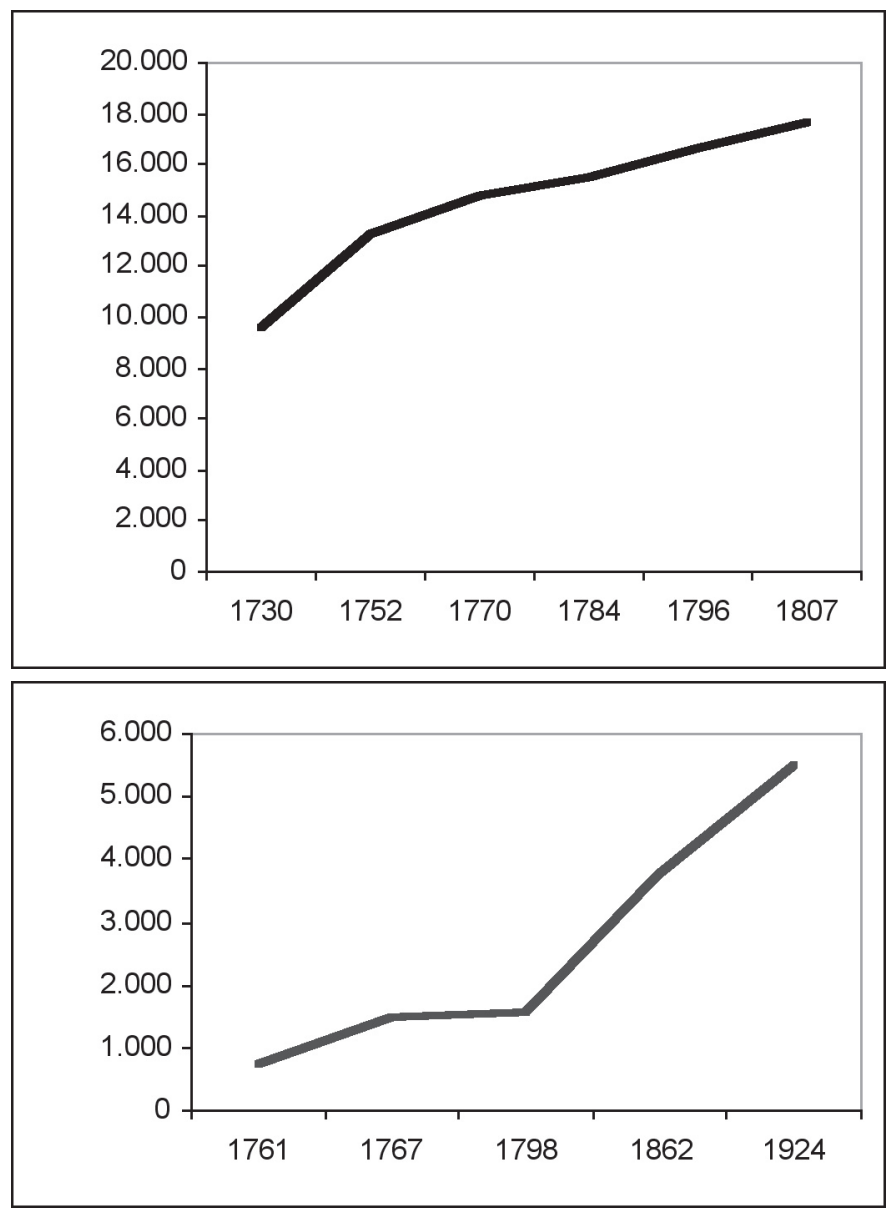

Figura 4a (superior): evolución de la superficie arrocera en el Reino de Valencia entre 1730 y 1807 (elaboración propia a partir de Mateu, 1987: 50).

FIgURA 4b (inferior): superficie transformada dentro de los límites de la Albufera (1761-1924) según Sanchis Ibor (2001: 269) (elaboración propia).

paludismo² $^{2}$ El estudio de Mateu (1987), demuestra cómo el saldo vegetativo de poblaciones pequeñas de la Ribera del Xúquer — donde los controles sanitarios eran menores - resultaba negativo. La sobremortalidad, derivada de las condiciones de insalubridad y de la alta morbilidad producida por la malaria, se contrarrestaba con un importante flujo inmigratorio, atraído por la demanda de brazos para la siembra y recolección del arroz.

2 Por ejemplo, en 1807 Tomás de Otero, miembro de la Real Sociedad Económica de Valencia, se refería a esta causa como una de las principales diferencias entre los arrozales asiáticos, donde «los indios connaturalizados en el contacto de aquellas miasmas no sienten sus efectos», y los valencianos, donde esto no se lograba por el importante flujo inmigratorio hacia las poblaciones arroceras. Archivo de la Real Sociedad Económica de Amigos del País de Valencia, 1807, C-47, I Agricultura, N. 9. 


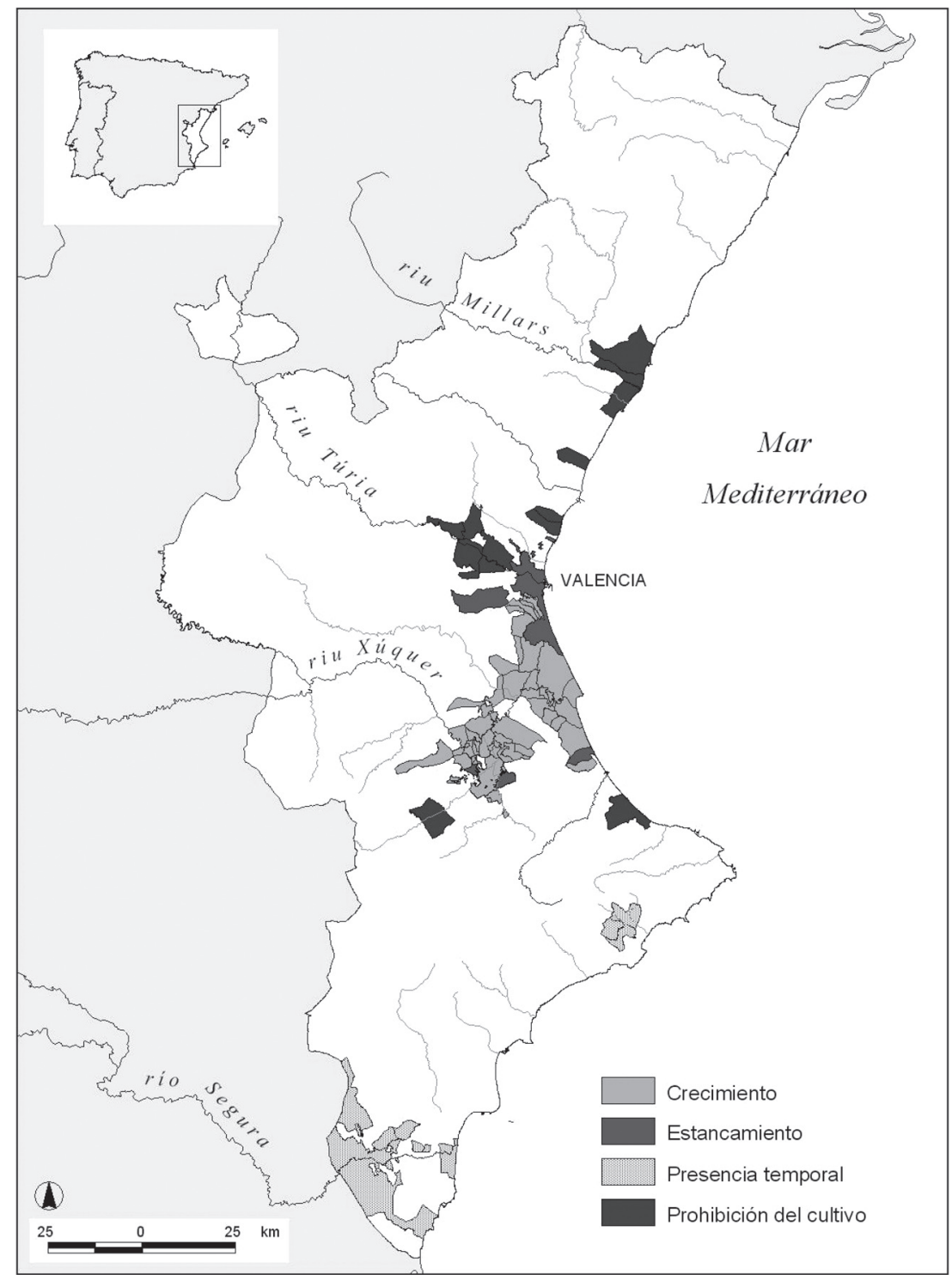

Figura 5. Presencia del arroz en tierras valencianas entre 1769 y 1787. Elaboración propia.

\subsection{Discursos de control de la enfermedad, percepción y acción sobre el medio}

Aunque la malaria ya era un importante problema de salud pública, la gran epidemia de la década de 1780 supuso un incremento cualitativo de los discursos médico y político sobre las causas y el control de la enfermedad, también de la relación entre las condiciones climáticas y la medicina (Capel, 1999). Desde la óptica ambientalista, las estrategias de lucha contra el fenómeno infeccioso se centraron en políticas de intervención sobre el 
medio a diversas escalas. Fundamentalmente se trataba de medidas basadas en la gestión ambiental que, debido al elevado conocimiento epidemiológico alcanzado, presentan una gran similitud con las patrocinadas en la actualidad por la OMS, con el fin de reducir el contacto entre hombre, vectores y patógenos (Keiser et al., 2005).

Siguiendo con el ejemplo del Reino de Valencia y con esa correlación entre mosquito y miasma/efluvio, la Real Cédula de $1785^{3}$ resulta un referente fundamental, puesto que se trata de una medida adoptada directamente por el Rey para evitar la presencia de aguas estancadas. De esta forma, se pretendía acabar de forma temporal con unas condiciones favorables para los miasmas, derivadas de unas condiciones climáticas excepcionales y de la presencia de arrozales, humedales naturales y, también, encharcamientos procedentes de una mala gestión hidráulica (fugas de acequias, evacuación deficiente de aguas negras, etc.). Sin embargo, su promulgación tuvo lecturas de mayor entidad, en la línea del trauma colectivo que estaba significando la epidemia en toda España.

En tierras valencianas, el grave problema epidemiológico que se había reproducido en multitud de pueblos con los arrozales, ambientalmente perjudiciales y económicamente muy rentables, incrementó el número de voces que exigían medidas permanentes y a medio plazo, tendentes a prohibir el cultivo del arroz y a desecar grandes extensiones de humedales mediante procesos de bonificación. Los proyectos para desecar estos últimos espacios se multiplicaron en las décadas finales del siglo XVIII (p.e. Gandia, Torreblanca u Oropesa), dentro del creciente interés, mostrado por parte de gobernantes, terratenientes y tratadistas de la Ilustración, por aunar salud pública y expansión de la agricultura. En Valencia, sin embargo, dicha postura chocó frontalmente con el potente interés arrocero, hasta el punto de que las medidas adoptadas por administraciones regionales (Capitán General, Real Audiencia de Valencia) y las voces de reputados científicos como Cavanilles, sucumbieron a las decisiones del Consejo de Castilla (Madrid), principal órgano de la monarquía absolutista.

Una muestra de la percepción de la estrecha relación entre la malaria y el arroz se encuentra en los acotamientos de arrozales, las prohibiciones parciales del cultivo y los censos de superficie que, promovidos por la administración valenciana, se multiplicaban inmediatamente después de una epidemia. Tras la producida en la década de 1780, además de los intentos por llevar a cabo este tipo de acciones, se inició la elaboración del Expediente General de Arroces, documento muy voluminoso y reservado únicamente a los asuntos prioritarios de la Corona (Memorial, 1803). El documento fue recogiendo, a su vez, los testimonios de científicos, médicos, comisionados, párrocos y particulares, relativos a las condiciones ambientales del óvalo central valenciano. A partir de esta extensa polémica, se alcanzaron altas cotas de reflexión científica en torno al origen natural o artificial de los espacios húmedos (Giménez-Font, 2008) y en torno a las relaciones entre la sociedad del momento y la enfermedad, utilizando incluso estadísticas demográficas simples para cuantificar el impacto del paludismo sobre las poblaciones arroceras (p.e., Cavanilles, 1795-1797; Memorial, 1803).

Junto a los ambiciosos planes de desecación y, por tanto, de modificación ambiental de aquellas zonas consideradas endémicas, existieron intentos de promover medidas de higiene pública y conductas de protección entre la población (Peset y Peset, 1972a; Riera, 1980). Los avances eran muy escasos y entre los remedios preservativos generales había

3 Real Cédula de S.M. y señores del Consejo, por la qual se manda que para evitar en lo sucesivo las Epidemias de Tercianas ocurridas en el Reyno de Valencia, se pongan en curso las Aguas estancadas, y se observen las demas reglas que se prescriben para dicho fin. Año 1785. Archivo Histórico Nacional, Diversos_Reales Cédulas, $\mathrm{n}^{\mathrm{o}} 711$. 
uno indiscutible, que resumía el doctor Llansol (1797: 179): «huir en quanto se pueda de tragar estos hálitos». No obstante, en su condición de buenos epidemiólogos, los médicos consiguieron, aún desconociendo la verdadera etiología de la enfermedad, ofrecer consejos tan afinados como el del mencionado Llansol: «conviene pues que por las mañanas, y noches, en que hay mas porcion de efluvios en la atmosfera, hasta que el calor del Sol los enrarezca, huir en quanto se pueda de ellos, lo que se hace levantándose más tarde en estos lugares, y acostandose un poco mas temprano. Ya las gentes han adoptado en muchos pueblos el uso de mosquiteras, con que se defienden por la noche de esta plaga, para poder tomar el sueño. [...] Conviene también muchísimo dormir en habitaciones de altos, poniendo [...] vidrieras á las ventanas que mas baten los aires, y procurando dar entrada, quando ya se ha levantado el Sol, y lograr la ventilación con menos riesgo» (Llansol, 1797: 179-180).

\section{Discusión y conclusiones}

Los resultados preliminares de esta investigación, pretenden destacar el interés de los estudios paleoepidemiológicos desde una perspectiva transversal, en la que la estructura geográfica de una epidemia histórica (Hagget, 2000) permita completar el esquema inicial sobre las causas de proliferación vectorial. De esta forma, el análisis histórico de procesos infecciosos bien documentados, como es el caso de la epidemia de 1783-1786 en España, pueden ofrecer claves interpretativas para la comprensión de algunos comportamientos del presente.

Para ello, hay que considerar que, aunque la etiología de la malaria no era correcta, los médicos y otros científicos del momento demostraron, en relación con el paludismo, que eran excelentes epidemiólogos. Parte de las soluciones de control ambiental planteadas en aquel momento mantienen una vigencia absoluta y las reflexiones de muchos médicos, con gran influencia en las políticas sanitarias, señalan las causas de la expansión en la conjunción de unas condiciones atmosféricas excepcionales, la introducción de nuevos cultivos, el incremento de la población en determinadas áreas y unas condiciones sociosanitarias deficientes.

Una de las cuestiones más relevantes en el desarrollo de la epidemia deriva de la posible influencia de una oscilación climática caracterizada por un aumento en la frecuencia de sequías e inundaciones. Cualitativamente, numerosos testimonios procedentes de la medicina demuestran una relación clara entre inviernos o primaveras lluviosas / veranos cálidos y secos con la enfermedad durante la década de 1780. Pese a la ausencia de análisis de mayor profundidad para el conjunto de España, diversos estudios de climatología histórica ya han verificado cuantitativamente la presencia de un período de gran inestabilidad, especialmente en el caso concreto de Cataluña, territorio afectado por la epidemia. La consideración de esta variable histórica cobra mayor interés si la incluimos en modelos predictivos de cambio climático, que consideran el factor epidemiológico entre las amenazas a medio plazo. Aunque las proyecciones todavía no son fiables, la modelización del clima es cada vez más precisa. Los recientes escenarios planteados para España (VVAA, 2007) se centran en los posibles cambios en la alteración y frecuencia de eventos climáticos extraordinarios (concentración de la precipitación, aumento de las olas de calor estivales), en condiciones, a priori, parecidas a las descritas para la Oscilación Maldá (Barriendos y Llasat, 2003). En ambos casos las consecuencias inmediatas repercuten en una alteración de ciertos ecosistemas y, en relación con esto, del comportamiento de enfermedades transmitidas por artrópodos. Algunos escenarios manejados por epidemiólogos (López-Velez y Molina, 2005) plantean la posibilidad de que modificaciones en la temperatura o la precipitación condicionen la tasa de crecimiento de ciertos vectores o faciliten la expansión y establecimiento de mosquitos 


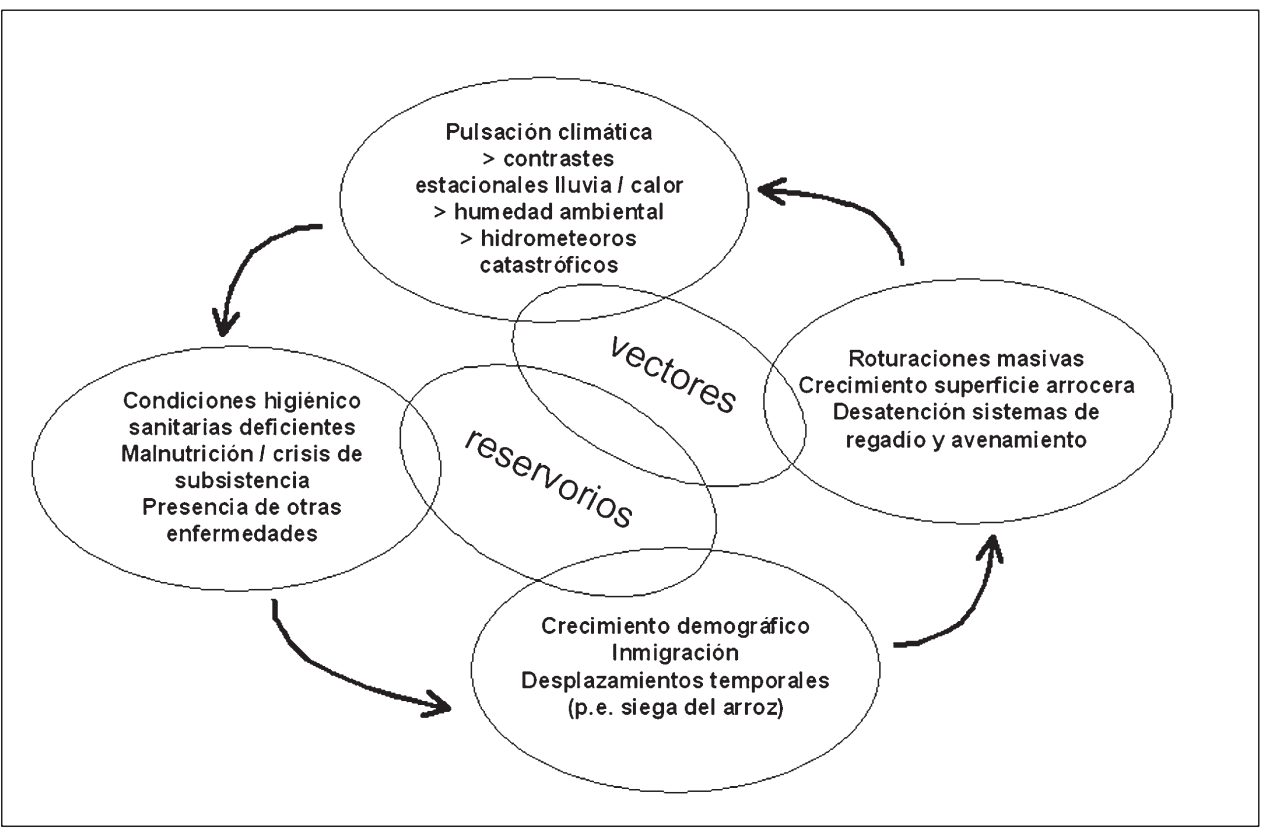

FiguRA 6. Esquema interpretativo aplicado a la epidemia de 1783-1786. Elaboración propia.

mucho más efectivos y peligrosos debido a su antropofília (p.e. Anopheles labranchiae y Anopheles sacharovi).

Estas hipótesis prospectivas enlazan con las planteadas para epidemias ocurridas en el pasado, como la que nos ocupa. Sin embargo, las enfermedades transmitidas por artrópodos revisten una gran complejidad por la interferencia de condiciones ambientales y sociales que, además, intervienen a distintos niveles jerárquicos. Este hecho requiere acercamientos transdisciplinares que permitan trabajar desde los aspectos geoclimáticos a las condiciones particulares de la población afectada. En este sentido, los estudios históricos pueden facilitar un mayor conocimiento del comportamiento de la enfermedad ante determinados cambios ambientales y sociales. Las notas preliminares aplicadas a parte del territorio valenciano, pretenden incidir en las posibilidades de análisis más detallados sobre espacios donde la disponibilidad de documentación y los estudios previos son abundantes.

Aunque la comparativa con la actualidad no es válida en estas mismas zonas, los resultados pueden ser extrapolables a otros espacios del planeta que repiten patrones parecidos a los de la sociedad española del Antiguo Régimen: economía de base agraria, crecimiento demográfico/afluencia de inmigrantes, problemas de desnutrición, condiciones higiénicas precarias, política asistencial muy deficiente e importantes cambios de usos del suelo relacionados con la deforestación y la gestión inadecuada del agua.

\section{Agradecimientos}

El autor quiere agradecer a Mariano Barriendos (Universitat de Barcelona) las consideraciones realizadas sobre el borrador. Esta investigación se inscribe dentro del Grupo de Investigación en Historia y Clima de la Universidad de Alicante (VIGROB187). 


\section{Bibliografía}

ALBEROLA, A. (2006): «Entre la sequía y la inundación. Una aproximación a las avenidas históricas de los ríos valencianos durante el siglo XVIII» en Chastagnaret, G. y Gil Olcina, A.: Riesgos de inundaciones en el Mediterráneo occidental. Casa de Velázquez-Universidad de Alicante, Madrid, pp. 1-30.

ALBEROLA, A. y BERNABÉ, D. (1999): «Tercianas y calenturas en tierras meridionales valencianas: una aproximación a la realidad médica y social del siglo XVIII», en Revista de Historia Moderna, $\mathrm{n}^{\mathrm{o}}$ 17, pp. 95-112.

BARBIERI, A.F.; OYA, D.; SILVEIRA, B. (2005): «Population and Land Use Effects on malaria Prevalence in the Southern Brazilian Amazon», en Human Ecology, no 33-6, pp. 847-874. http:// www.springerlink.com/content/p12557331t4365n2/fulltext.pdf (consulta abril de 2008).

BARRIENDOS, M. y LLASAT, C. (2003): «The case of the 'Maldá' anomaly in the western mediterranean basin (AD 1760-1800): an example of a strong climatic variability» en Climatic Change, $\mathrm{n}^{\circ}$ 61, pp. 191-216. http://www.springerlink.com/content/m700123435167665/fulltext. pdf (consulta marzo de 2007).

BARRIENDOS, M. y MARTÍN-VIDE, J. (1998): «Secular Climatic Oscillations as Indicated by Catastrophic Floods in the Spanish Mediterranean Coastal Area (14th-19th Centuries)» en Climatic Change, $\mathrm{n}^{\mathrm{o}} 38$, pp. 473-491. http://www.springerlink.com/content/m7m4482048p623k5/fulltext. pdf (consulta abril de 2008).

CAPEL, H. (1999): «Medicina y clima en la España del siglo XVIII» en Revista de Geografía, no 32-33, pp. 79-105.

GIMÉNEZ-FONT, P. (2008): Las transformaciones del paisaje valenciano en el siglo XVIII. Una perspectiva geográfica. Edicions d'Alfons el Magnànim, València, 460 pp.

GUITEKO, AK.; LINDSAY, SW.; CONFALONIERI, U.; PATZ, JA.: «Climate chage and vectorborne diseases: a regional analysis» en Bull. World Health Organization, $\mathrm{n}^{\circ}$ 78, pp. 1136-1147. https://www.who.int/bulletin/archives/78(9)1136.pdf (consulta abril de 2008).

HAGGET, P. (2000): The Geographical Structure of Epidemics, Claredon Press, Oxford, 149 pp.

HAY, S.I.; GUERRA, C.A.; TATEM, A.J.; NOOR, A.M.; SNOW, R.W. (2004): «The global distribution and population at risk of malaria: past, present, and future» en The Lancet Infectious Diseases, $\mathrm{n}^{\circ}$ 4-6, pp. 327-336.

KEISER, J.; SINGER, B.H.; UTZINGER, J. (2005): «Reducing the burden of malaria in different eco-epidemiological settings with environmental management: a systematic review» en The Lancet Infectious Diseases, $\mathrm{n}^{\circ}$ 5-11, pp. 695-708.

KINGTON, J. (1988): The Weather of the 1780s over Europe, Cambridge University Press, Cambridge, $180 \mathrm{pp}$.

KLINKENBERG, E.; HUIBERS, F.; TAKKEN, W.; TOURE, Y.T. (2002): «Water management as a tool for malaria mosquito control? The case of the Office du Niger, Mali» en Irrigation and Drainage Systems, nº 16, pp. 201-212. http://www.springerlink.com/content/j7166v8616311428/ fulltext.pdf (consulta abril de 2008).

KOELLE, K.; RODÓ, X.; PASCUAL, M.; YUNUS, Md; MOSTAFA, G. (2005): «Refractory periods and climate forcing in cholera dynamics», en Nature 436,4, pp. 696-700. http://www.icddrb. org/images/Dr._Md_Yunus_Refractory_periods_and_climate_forcing_in_cholera_dynamics. pdf (consulta mayo de 2008).

LÓPEZ-VÉLEZ, R. y MOLINA, R. (2005) «Cambio climático en España y riesgo de enfermedades infecciosas y parasitarias transmitidas por artrópodos y roedores» Rev. Esp. Salud Publica ${ }^{\circ}$ 79-2, pp. 177-190.

http://scielo.isciii.es/scielo.php?script=sci_arttext\&pid=S1135-57272005000200006\&lng=es\&nr $\mathrm{m}=$ iso (consulta abril de 2008).

MATEU, E. (1987): Arroz y paludismo: riqueza y conflictos en la sociedad valenciana del siglo XVIII. Ed. Institució Alfons el Magnànim, València, 192 pp.

McMICHAEL, A.; WOODRUFF, E.; HALES, S. (2006): «Climate change and human health: present and future risks» en The Lancet, $\mathrm{n}^{\circ}$ 367, pp. 859-869.

McNEILL, W. (1984): Plagas y Pueblos, Siglo XXI, Madrid, 303 pp. 
PÉREZ MEDINA, T. V. (1991): «Arròs, paludisme i població a la comarca de l'Horta. L'epidemia de 1784» en Afers, $n^{\circ} 11-12$, pp. 137-150.

PÉREZ MOREDA, V. (1980): Las crisis de mortalidad en la España interior (siglos XVI-XIX), Siglo XXI, Madrid, $526 \mathrm{pp}$.

PÉREZ MOREDA, V. (1984): «Crisis demográficas y crisis agrarias: paludismo y agricultura en España a fines del siglo XVIII» en Congreso de Historia Rural. Siglos XV al XIX. Universidad Complutense, Madrid, pp. 333-354.

PERIS, T. (2001): Història de la Ribera. L'escenari i els protagonistes, Ed. Bromera. Alzira, 256 pp.

PESET, M. y PESET, J.L. (1972a): «Cultivo del arroz y paludismo en la Valencia del siglo XVIII» en Hispania, $\mathrm{n}^{\circ} 121$, pp. 277-375.

PESET, M. y PESET, J.L. (1972b): Muerte en España. Política y sociedad entre la peste y el cólera. Ed. Seminarios y Ediciones, Madrid, 258 p.

REITER, P. (2001): «Climate change and mosquito-borne disease» en Environmental Health Pespective, no 109 (suppl 1), pp. 141-161. http://www.pubmedcentral.nih.gov/picrender.fcgi?artid= 1240549\&blobtype $=$ pdf $($ consulta abril 2008)

RIERA, J. (1980): José Masdevall y la medicina española ilustrada (enseñanza, epidemias y guerra a finales del siglo XVIII). Universidad de Valladolid, Valladolid, $257 \mathrm{p}$.

ROGERS, D.J. y RANDOLPH, S.E. (2000): «The Global Spread of Malaria in a Future, Warmer World», en Science, n $^{\circ} 289$, pp. 1763-1766.

www.sciencemag.org/cgi/reprint/289/5485/1763.pdf (consulta abril de 2008).

SANCHIS IBOR, C. (2001): Regadiu i canvi ambiental a l'Albufera de València. Universitat de València, València. $332 \mathrm{p}$.

URTEAGA, L. (1993): «La teoría de los climas y los orígenes del ambientalismo» en Geo Crítica, $\mathrm{n}^{\circ} 99,55 \mathrm{pp}$.

VV.AA. (2007): El cambio climático en España. Estado de situación. Documento resumen. Noviembre de 2007. www.mma.es/secciones/cambio_climatico/pdf/ad_hoc_resumen.pdf (consulta mayo de 2008)

ZHOU, G.; MINAKAWA, N.; GITHEKO, A.K.; YAN, G. (2004): «Association between climate variability and malaria epidemics in the East African highlands» Proceedings of the National Academy of Sciences of the U.S.A. (PNAS), $\mathrm{n}^{\circ}$ 101-8, pp. 2375-2380. www.pnas.org/cgi/doi/10.1073/ pnas.0308714100 (consulta abril de 2008)

Obras médicas y legislativas contemporáneas a la epidemia del siglo XVIII

CAVANILLES, A. J.(1795-1797): Observaciones sobre la Historia Natural, Geografía, Agricultura, Población y Frutos del Reyno de Valencia. Imprenta Real de Madrid, 2 vol. [Ed. facsímil, Biblioteca Valentina, 1975]

CIBAT, A. (1806): Memoria sobre el problema ¿por qué motivos ó causas las tercianas se han hecho tan comunes y graves en nuestra España?, ¿Con qué medios podría precaverse o destruirse?, Imprenta Real, Madrid, $122 \mathrm{pp}$.

DE ASED Y LATORRE, A. (1784): Memoria instructiva de los medios de precaver las malas resultas de un Temporal excesivamente húmedo, como el que se ha observado desde principios de Setiembre de 1783, hasta ultimos de Abril de 1784. Imprenta de Blas Miedes, Zaragoza, $30 \mathrm{pp}$.

FRANCO, V.I., (1797): Contextación a las observaciones sobre la necesidad de la cría de arrozes en la Ribera del Xúcar, Reyno de Valencia, e influencia de su cultivo en la salud pública, que publicó el Abate D. Josef Antonio Cavanilles. Imp. Tomás de Orga. Valencia, 52 pp.

GARCÍA BURUNDA, J. (1785): Informe del Real Proto-Medicato en que se proponen las observaciones médicas para indagar las causas, y método curativo de las tercianas, tomando noticias de los progresos de esta enfermedad en el presente año de 1785, que puedan servir de preservativo en adelante. Madrid. (cit. en Pérez Moreda, 1985: 353)

LLANSOL, F. (1797): Topografía médica general de la Ribera del Xúcar y particular de la villa de Alzira, trascrito en RIERA, J.y GRANDA-JUESAS, J. (1988).: Epidemias y paludismo en la Ribera del Júcar (una topografía médica del siglo XVIII). Ed. Universidad de Valladolid, pp. 63-224. 
MASDEVALL, J. (1786): Relación de la epidemia de Calenturas pútridas y malignas, que en estos últimos años se han padecido en el Principado de Cataluña; y principalmente de la que se descubrió el año pasado de 1783 en la Ciudad de Lérida, Llano de Urgel y otros muchos corregimientos y partidos, con el método feliz, pronto y seguro para curar semejantes enfermedades. Imprenta Real, Madrid, 136 p. [Ed. facsímil, Universidad de Valladolid, 1996].

MEMORIAL (1803): Memorial Ajustado del Expediente General que en virtud de reales ordenes se sigue en consejo pleno para consultar á S.M. sobre la utilidad ó perjuicio de la siembra de arroz en el Reyno de Valencia, y fixación de pueblos y Terrenos en que aquella pueda executarse. Madrid, Imprenta Real (1803).

REAL CÉDULA (1785): Real Cédula de S.M. y señores del Consejo, por la qual se manda que para evitar en lo sucesivo las Epidemias de Tercianas ocurridas en el Reyno de Valencia, se pongan en curso las Aguas estancadas, y se observen las demas reglas que se prescriben para dicho fin. Año 1785. Imp. Benito Monfort, Valencia, 5 pp.

TOVARES, J. (1786): Descripción histórico-médica, o memoria sobre la epidemia de calenturas intermitentes, observada en España en el año de 1786. Madrid (cit. en Pérez Moreda, 1985: 354)

TRONCOSO, M. (1785): «Memoria Físico-Médica sobre la epidemia de tercianas que este presente año se ha padecido en la Ciudad de Cordoba» en Memorial Literario, Madrid, vol. VI: 22, pp. 189-195. (cit. en Capel, 1999: 89).

VILLALBA, J. de (1803): Epidemiología española, o historia cronológica de las pestes, contagios, epidemias y epitozootias que han acaecido en España desde la venida de los cartagineses hasta el año 1801 [...], Imprenta de Mateo Repullés, Madrid, 2 vols. 
